\title{
Measuring Brain Variability Via Sulcal Lines Registration: A Diffeomorphic Approach
}

\author{
Stanley Durrleman ${ }^{1,2}$, Xavier Pennec ${ }^{1}$, Alain Trouvé ${ }^{2}$, and Nicholas Ayache ${ }^{1}$ \\ ${ }^{1}$ INRIA - Asclepios Research Project, Sophia Antipolis, France \\ ${ }^{2}$ Centre de Mathématiques et leurs applications, ENS de Cachan, Cachan, France
}

\begin{abstract}
In this paper we present a new way of measuring brain variability based on the registration of sulcal lines sets in the large deformation framework. Lines are modelled geometrically as currents, avoiding then matchings based on point correspondences. At the end we retrieve a globally consistent deformation of the underlying brain space that best matches the lines. Thanks to this framework the measured variability is defined everywhere whereas a previous method introduced by P. Fillard requires tensors extrapolation. Evaluating both methods on the same database, we show that our new approach enables to describe different details of the variability and to highlight the major trends of deformation in the database thanks to a Tangent-PCA analysis.
\end{abstract}

\section{Introduction}

Measuring brain variability among populations is a challenging issue. A very promissing way as emphasized in 12 for instance is based on statistical analysis of brain deformations computed thanks to registrations of images or geometrical primitives like cortex surfaces, sulcal lines or landmarks. In the case of primitives, however, the registrations are rarely based on a consistent geometrical modelling of both primitives and deformations. We follow here the approach introduced in [3] and [4]: we model sulcal lines as currents. Such currents are then considered as 'geometrical landmarks' that guide a globally consistent deformation of the underlying biological material. After presenting this registration framework in section 2. we apply the method to a database of sulcal lines and deduce statistical measures of brain variability within the studied population in section 3 . Eventually we show how the underlying geometrical modelling makes the measured variability different from this obtained in [5] on the same dataset but in a different framework (called here FAPA's), and how it enables to obtain new statistical results which may lead to new scientific findings.

\section{Registering Lines Sets}

Registering a lines set $L_{0}$ onto another $L_{1}$ consists in looking for the most regular deformation $\phi$ that acts on $L_{0}$ and best matches $L_{1}$. More precisely, we follow the approach proposed in [3]: the unknown deformation is searched in a subgroup of diffeomorphisms of the space $\mathbb{R}^{3}$ and the lines are modelled as currents. We find

N. Ayache, S. Ourselin, A. Maeder (Eds.): MICCAI 2007, Part I, LNCS 4791, pp. 675 682, 2007.

(C) Springer-Verlag Berlin Heidelberg 2007 
the registrations thanks to the minimization of a cost function which makes a compromise between the regularity of the deformation and the fidelity to data.

\subsection{Lines Modelled as Currents}

The space of currents is a vector space which can be equipped with a norm that enables to measure geometrical similarity between lines. In this space, lines could be discrete or continuous and consists in several different parts. All these objects are handled in the same setting and inherit from many interesting mathematical properties: linear operations, convergence, etc.. Moreover, the distance between lines does not make any assumptions about point correspondences, even implicitly. This framework differs therefore from usual methods like in [6] where lines are considered as unstructured points set and "fuzzy" correspondences assumed.

We restrict now the discussion to what is needed in the following and we refer the reader to [7] and [3] for more details on the theory. We call here a line set $L$ a finite collection of $n$ continuously differentiable mappings $L_{i}:[i / n,(i+1) / n] \rightarrow$ $\mathbb{R}^{3}: L=\cup_{i=0}^{n-1} L_{i}$. This formulation is compatible with discrete set of lines, each line $L_{i}$ being given by $n_{i}$ samples and $\left(n_{i}-1\right)$ segments $\left[s_{k}^{i}, s_{k+1}^{i}\right]_{1 \leq k \leq n_{i}-1}$.

A vector field $\omega$ is a differentiable mapping that associates to every points $x \in \mathbb{R}^{3}$ a vector $\omega(x) \in \mathbb{R}^{3}$. Let us denote $W$ a linear space of vector fields. Our space of currents $W^{*}$ is defined as the set of the continuous linear mappings from $W$ to $\mathbb{R}$. A line set $L$ can thus be seen as a current thanks to the formula:

$$
\forall \omega \in W, \quad L: \omega \longrightarrow \int_{0}^{1}<\omega\left(x_{t}\right), \tau\left(x_{t}\right)>_{\mathbb{R}^{3}} d t
$$

where $\tau$ is the unit tangent vector (defined almost everywhere) of the line set $L$ at point $x_{t}$. For $W$ we choose a reproducing kernel Hilbert space (r.k.h.s.) whose kernel $K^{W}$ is isotropic and Gaussian (for more details on r.k.h.s, see [8]). This induces a norm on the space of currents that is computable in case of discrete lines. Indeed, when the line $L$ is sampled, it can be approximated in $W^{*}$ as the segments length tends to 0 by the sum of Dirac currents at the points $\left(c_{i}\right)$ centers of the segments $\left[s_{i}, s_{i+1}\right]: L=\sum_{k} \delta_{c_{k}}^{\tau_{k}}$ where $\tau_{k}=s_{k+1}-s_{k}$. (for all vector field $\omega, \delta_{x}^{\alpha}(\omega)=\langle\omega(x), \alpha\rangle_{\mathbb{R}^{3}}$ : a Dirac current can be seen as a vector $\alpha$ concentrated at one point $x)$. The Hilbertian inner product between $L=\sum_{i=1}^{n-1} \delta_{c_{i}}^{\tau_{k}}$ and $L^{\prime}=\sum_{i=1}^{m-1} \delta_{c_{i}^{\prime}}^{\tau_{k}^{\prime}}$ where $n$ is not necessarily equal to $m$ is then given by: $\left\langle L, L^{\prime}\right\rangle_{W^{*}}=\sum_{i=1}^{n-1} \sum_{j=1}^{m-1}\left\langle\tau_{i}, K^{W}\left(c_{i}, c_{j}^{\prime}\right) \tau_{j}^{\prime}\right\rangle_{\mathbb{R}^{3}}$ where for all points $x, y$, $K^{W}(x, y)=g(x-y)$ Id and $g$ is a Gaussian function. The distance between two lines is then given by: $d^{2}\left(L, L^{\prime}\right)=\left\|L^{\prime}-L\right\|_{W^{*}}^{2}=\|L\|_{W^{*}}^{2}+\left\|L^{\prime}\right\|_{W^{*}}^{2}-2\left\langle L, L^{\prime}\right\rangle_{W^{*}}$. This converges to the distance between two continuous lines when the segments lengths tend to zero. Eventually, this distance used as a fidelity to data term will prevent from systematically overfitted registrations.

\subsection{Diffeomorphic Registration}

We use here the large deformation framework founded in the paradigm of Grenander's group action approach for modelling objects (see 941011]). This 


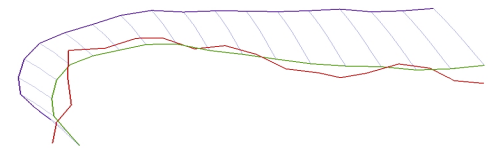

Fig. 1. Lines registration using an approach based on currents. Dark blue line is transported to the green line that matches the red line. The distance between red and green lines (i.e the precision of the matching) is computed although the sampling of each line is different, in particular no point to point correspondence is imposed.

framework enables to find a globally consistent deformation of the underlying space that best matches the lines sets. This differs from 5 where each line is registered individually without assuming spatial consistency of the displacement field. The global constraint as well as the introduction of a fidelity to data term lead to residual matching errors (the distance between red and green lines in figure 11) considered here as noise. In [5] denoising and matching are two separate processings.

The considered deformations are diffeomorphisms, solutions $\phi_{1}^{v}$ at time $t=1$ of the flow equation: $\frac{\partial \phi_{t}}{\partial t}=v_{t} \circ \phi_{t}$ and $\phi_{0}=\mathrm{id}_{\mathbb{R}^{3}}$. The tangent vector field $v_{t}$ at each time $t$ belongs to a r.k.h.s. $V$ with kernel $K^{V}$ that controls the regularity of the final diffeomorphisms. The induced norm defines in turn a distance between $\phi$ and the identity: $d_{V}^{2}(\mathrm{id}, \phi)=\inf \left\{\int_{0}^{1}\left\|v_{t}\right\|_{V}^{2} \mid v \in L^{2}([0,1], V), \phi=\phi_{1}^{v}\right\}$.

In order to register a line onto another we define $\phi . L$ the action of a diffeomorphism $\phi$ on a current $L$ by: $\phi \cdot L(\omega)=L(\phi \cdot \omega)$ and $\phi \cdot \omega(x)=\left(d_{x} \phi\right)^{t} \omega(\phi(x))$ for all vector fields $\omega$. This action coincides with the geometrical transportation of lines. In particular $\phi . \delta_{x}^{\alpha}=\delta_{\phi(x)}^{d_{x} \phi(\alpha)}$.

Our registration problem is to map a set of $n$ labelled sulcal lines $L_{0}=$ $\cup_{i=1} L_{0, i}$ to another labelled set $L_{1}=\cup_{i=1} L_{1, i}$. It is then reduced to the search of a family of vector fields $v: t \in[0,1] \longrightarrow v_{t}$ that minimizes the following cost function $J: J(v)=\gamma d_{V}^{2}\left(\mathrm{id}, \phi_{1}^{v}\right)+\sum_{i=1}^{n}\left\|\phi_{1}^{v} \cdot L_{0, i}-L_{1, i}\right\|_{W^{*}}^{2}$ where $\gamma$ controls the importance of the regularity against the fidelity to data.

It has been shown (in [4 for instance) that such an optimal diffeomorphism always exists, that its path defined by $\phi_{t}^{v}$ for the optimal $v$ is geodesic with respect to the distance $d_{V}$ and is an interpolation via the kernel $K^{V}$ of the trajectories of the samples that numerically define the lines. Moreover we can show that one can recover the trajectory of any points of the space knowing only the initial speed at each lines samples. This means that a minimizing diffeomorphism is entirely determined by a finite set of parameters. This dramatic dimensionality reduction is of great importance to define statistics on deformations.

\subsection{Experiments and Results}

Through the Asclepios-LONI associated team Brain-Atlas we used the same dataset as in [5] of cortical sulcal landmarks (72 per brain) delineated in every subject scanned with 3D MRI (age: $51.8+/-6.2$ years). In order to compare our measures of variability, we used the same set of 72 mean lines as in [5]. For 34 
subjects in the database, we registered the mean lines set onto every subject's lines set. We computed the registrations thanks to J. Glaunès' algorithm detailed in 3. We manually set $\gamma=0.1$ and the standard deviation of $K^{V}$ and $K^{W}$ respectively to $\sigma_{V}=25 \mathrm{~mm}$ and $\sigma_{W}=5 \mathrm{~mm}$. The diameter of the brains is about $120 \mathrm{~mm}$. For every subject deformations, we store the initial speed vectors of the mean lines samples that completely parametrize the deformation.

\section{Statistics on Deformations}

To do statistics, we take advantage of a tangent space representation like in 12 or 13 in case of finite dimensional manifolds. The deformations are indeed completely determined by their initial speed vector field that belongs to the linear space $V$ provided by an Hilbertian norm \|\|$_{V}$. We recall that such a dense vector field is in turn parametrized by the finite set of initial speed vectors on the mean lines samples: $\left\{\left(v_{0, k}^{s}\right)\right\}, 1 \leq k \leq N$ where $N$ is the total number of mean lines samples, and that we stored these vectors for each of the 34 registrations. Statistics on deformations are then reduced to statistics in $\mathbb{R}^{3 N}$ where the norm of the vector is the norm in $V$ of its associated dense vector field. Our study is focused on variance which measures how locally the space is deforming and covariance which measures the correlations between different points trajectories.

Our results are then compared to those obtained by FAPA in [5] where the statistics are based on the mean lines samples displacement field computed from a point-to-point registration algorithm.

\subsection{Variance of Deformations}

At each mean lines sample $k_{0}$ we compute the empirical covariance matrix from the 34 initial speed vectors $\left(v_{0, k_{0}}^{s} \in \mathbb{R}^{3}\right.$ for each subject $\left.s\right)$. These $3 \times 3$ matrices are represented as ellipsoids like in figure 2. They show how locally one point is varying among the studied population. On the other hand, thanks to the diffeomorphic approach, we can compute the tangent vector at each point of a 'mean brain surface' and hence the empirical covariance matrix of the deformations at those points. Figure 3 and 4 show such a surface where each point was coloured according to the 3D rms norm of the covariance matrix. In FAPA's method matrices computed on mean lines samples are downsampled and then extrapolated in the whole space thanks to a log-Euclidian framework ([1314). Comparing the results of both approaches highlights the different hypothesis made to model the lines, to remove noise and to extrapolate the variability to the brain surface.

Regularity of the Variability: The figure 2 shows that the point matching method of FAPA leads to irregular tensor fields at lines extremities and between lines whereas our global regularity constraint makes the retrieved variability spatially smoother. In our approach we leave aside the variability contained in the residual matching errors considered as noise. In FAPA's work the variability is denoised afterwards by removing extremal large tensors before the extrapolation. 


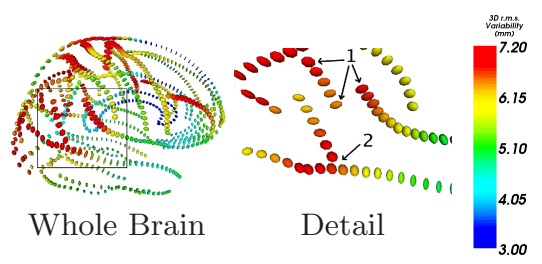

a - Our approach

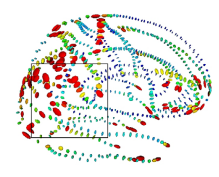

Whole Brain

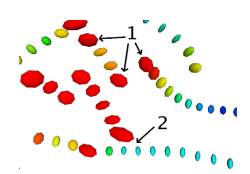

Detail

b - FAPA's method

Fig. 2. At each sampling point, ellipsoids represent the square root of the empirical covariance matrix of the initial speed vectors (left hand side) or displacement field (right hand side). In FAPA's method, extremal points are supposed to be matched: this induces a high variability at lines extremities (area 1, right). This is avoided by the current approach (area 1, left). In FAPA's method each line is registered individually: the variance can vary dramatically at lines crossing (area 2, right). Our global regularity constraint leads to smoother results (area 2, left).

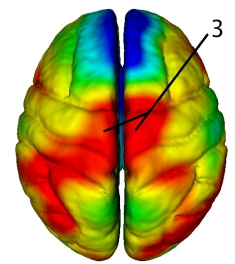

Variability map Covariance matrices a - Our approach

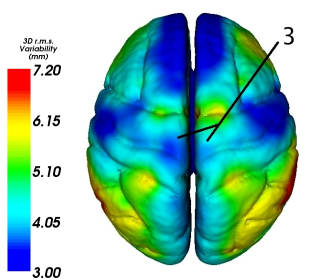

Variability map Covariance matrices b - FAPA's method

Fig. 3. On the variability maps, a tangential variability is retrieved in area 3 (extremities of central sulci) by our method and not by FAPA's one. The covariance matrices in this region show that the variability is mainly longitudinal. Since in FAPA's work large extremal tensors are removed before the extrapolation, the tangential variability is not captured and the total variability is small.

Tangential Variability: One major drawback of FAPA's method as underlined in [5] is the systematically under-estimated tangential variability. This aperture problem is particularly visible on the top of the brain as shown in figure 3. Our approach enables to find a larger part of this variability which is, as we will see, one of the major variation trends within the sample (cf fig. 6). Otherwise, the non-tangential part is in relative good agreement in most parts.

Distinction between correlated and anticorrelated motions: In our approach the deformation field is extrapolated before computing the covariance matrix. By contrast in FAPA's method the matrices are extrapolated without assuring that the extrapolated tensors derive from an underlying deformation. As shown figure 5 this difference theoretically enables in our case to distinguish between areas where samples are moving in a correlated or anti-correlated manner. This is a possible explanation of the different variability maps retrieved in 

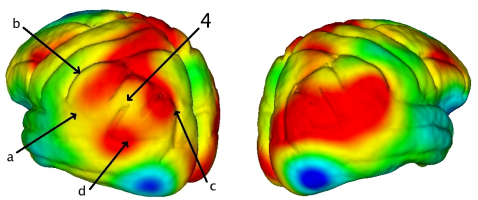

Left Hemisphere Right Hemisphere a - Our approach

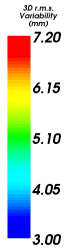

Left Hemisphere Right Hemisphere

b - FAPA's method

Fig. 4. Area 4 is surrounded by 4 major sulci: sylvius fissure (a), postcentral sulcus (b), intraparietal sulcus (c) and superior temporal sulcus (d). On the left hemisphere the first two move mostly in a decorrelated manner with respect to the last two sulci whereas their respective motions are much more correlated on the right hemisphere. Our approach tries to combine the motion of all lines and therefore leads to a small variability in area 4 on the left hemisphere and to a large one on the right hemisphere. With FAPA's method this asymmetry is not retrieved directly.
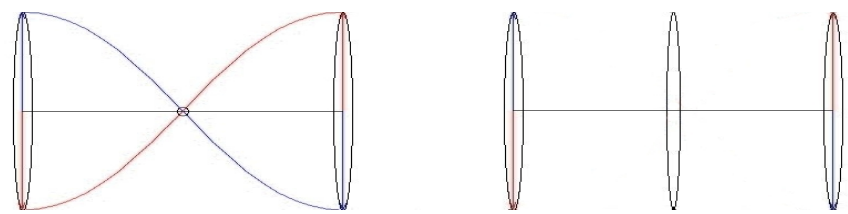

Fig. 5. Extrapolation schemes in the simple case of anti-correlated vectors. Right: In FAPA's framework the tensors are computed at the samples points and then extrapolated in the middle point: the tensor in the middle is similar to the two others. Left: In our approach we first extrapolate the vector field and then compute at each point the covariance matrix. Since the vectors are anti-correlated, the field is close to zero at the center and the variability measured at this point is negligible.

area 4 of figure 4 . Note that the asymmetry we found between left and right hemispheres was also retrieved in other recent studies 15.

\subsection{Principal Modes of Deformation}

Let us see the field $\left\{v_{0, k}^{s}\right\}$ for each subject $s$ as a unique vector in $\mathbb{R}^{3 N}$ provided by the norm \|\|$_{V}$, (the norm of its associated dense vector field). We then carry out a Principal Component Analysis (PCA) on these vectors with respect to the given norm (e.g. the first principal mode is given by $\operatorname{argmax}_{v \neq 0} \frac{\sum_{s}\left\langle v_{0}^{s}, v\right\rangle_{V}^{2}}{\|v\|_{V}^{2}}$ ). This analysis enables to take into account the global correlations of all points motion together and synthesizes the main trends of deformation in the database.

Given a mode $\tilde{v} \in V$, we can compute the unique geodesic deformation process in the sense of the distance $d_{V}$ whose initial tangent vector field is $\tilde{v}$. The eigenvectors $\tilde{v}$ were normalized $\left(\|\tilde{v}\|_{V}=1\right)$ so that the deformations are seen 

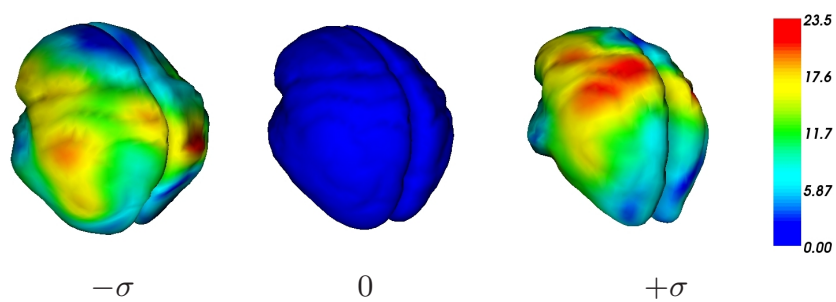

Fig. 6. First Mode of Deformation obtained by a PCA on initial vector speed fields. Original mean brain surface (Center) and its deformation at $-\sigma$ (Left) and $+\sigma$ (Right). Colours measure the displacement of each point along the deformation process (in $\mathrm{mm}$ ).

between $[-\sigma, 0]$ for shooting the vector $-\tilde{v}$ and $[0, \sigma]$ for $\tilde{v}$. Results are shown figure 6. Movies of deformation are available at first author's webpag 1 .

\section{Conclusion and Perspectives}

The framework presented here provides a consistent geometrical framework for measuring brain variability from sulcal lines registration. The lines are considered as geometrical objects and not as sets of landmarks, which means that we completely avoid computing point correspondences. A computable distance is defined on these lines that enables to include a noise model on lines within the framework. Although the optimal matching is parametrized with a finite set of parameters, the deformation field returned is dense, enabling to analyze the brain variability outside the data on the basis of an explicit deformation modelling. These three steps: denoising, lines matching and extrapolation are handled here consistlently in the same setting whereas they often lead to separate processings like in FAPA's work. This enables to give alternatives to some of the major drawbacks of other methods like the aperture problem for instance. The method is also generative: we can define an arbitrary deformation and hence generate deformed lines, illustrating thus the variability the method captured and highlighting the major trends of deformation within the database via a tangent-PCA analysis. Finally the approach is not limited to lines but could also be applied directly to register surface meshes (like in [16]) or images.

Besides such methodological advantages, the retrieved variability which differs from [5] are still to be validated by anatomical interpretations and by the model's predictive capability. At this stage, actually, the qualitative comparison mainly emphasizes the different hypothesis on which each model is based. Since our diffeomorphic constraint may be considered sometimes as too restrictive, one could define refinements that take into account possible local non diffeomorphic variations between subjects. Eventually, our new findings have to be confirmed by applying the method to other datasets of sulcal landmarks ([17]) or to other features that may be more relevant in an anatomical point of view. The integrative

${ }^{1}$ http://www-sop.inria.fr/asclepios/personnel/Stanley.Durrleman/ 
capability of the method could help actually to define a general model of brain variability based on multiple sources of input.

Acknowledgments We would like now to thank Paul Thompson (Lab of NeuroImaging, UCLA School of Medicine) through the Asclepios-LONI associated team for providing the dataset of sulcal lines, Joan Glaunès (Lab. de Mathématiques Appliquées, Université Paris 5) whose code was used to compute the registrations and Pierre Fillard (INRIA-Asclepios) for giving access to his results. Images and movies were made using the software MedINRIA2 This work was partly supported by the European IP projet Health-e-Child (IST-2004-027749).

\section{References}

1. Ashburner, et al.: Identifying global anatomical differences: deformation-based morphometry. Human Brain Mapping 6(5-6), 348-357 (1998)

2. Chung, M., et al.: A unified statistical approach to deformation-based morphometry. NeuroImage 18, 595-606 (2001)

3. Glaunès, J.: Transport par difféomorphismes de points, de mesures et de courants pour la comparaison de formes et l'anatomie numérique. $\mathrm{PhD}$ thesis, Université Paris 13 (2005), http://cis.jhu.edu/ joan/TheseGlaunes.pdf

4. Miller, M., Trouvé, A., Younes, L.: Geodesic shooting for computational anatomy. Journal of Mathematical Imaging and Vision (2006)

5. Fillard, P., Arsigny, V., Pennec, X., Hayashi, K., Thompson, P., Ayache, N.: Measuring brain variability by extrapolating sparse tensor fields measured on sulcal lines. NeuroImage 34(2), 639-650 (2007)

6. Chui, H., Rangarajan, A.: A new point matching algorithm for non-rigid registration. Comput. Vis. Image Underst. 89(2-3) (2003)

7. Vaillant, M., Glaunès, J.: Surface matching via currents. In: Christensen, G.E., Sonka, M. (eds.) IPMI 2005. LNCS, vol. 3565, Springer, Heidelberg (2005)

8. Aronszajn, N.: Theory of reproducing kernels. Trans. Amer. Math. Soc. (68) (1950)

9. Grenander, U.: General pattern theory. Oxford Science Publications (1993)

10. Glaunès, J., Joshi, S.: Template estimation from unlabeled point set data and surfaces for computational anatomy. In: Proc. of Workshop MFCA (2006)

11. Marsland, S., Twining, C.: Constructing diffeomorphic representations for the groupwise analysis of non-rigid registrations of medical images. T.M.I (2004)

12. Vaillant, M., Miller, M., Younes, L., Trouvé, A.: Statistics on diffeomorphisms via tangent space representations. NeuroImage 23, 161-169 (2004)

13. Pennec, X., Fillard, P., Ayache, N.: A Riemannian framework for tensor computing. International Journal of Computer Vision 66(1), 41-66 (2006)

14. Arsigny, V., Fillard, P., Pennec, X., Ayache, N.: Log-Euclidean metrics for fast and simple calculus on diffusion tensors. M.R.M. 56(2), 411-421 (2006)

15. Fillard, P.: al: Evaluating brain anatomical correlations via canonical correlations analysis of sulcal lines (In: Stat. Registration and atlas formation)

16. Vaillant, M., Qiu, A., Glaunès, J., Miller, M.: Diffeomorphic metric surface mapping in subregion of the superior temporal gyrus. NeuroImage 34(3) (2007)

17. Mangin, J.F., et al: Object-based morphometry of the cerebral cortex. IEEE Trans. Med. Imag. (2004)

$\overline{{ }^{2} \text { http://www-sop.inria.fr/asclepios/software/MedINRIA/ }}$ 